\title{
Téoros
}

Revue de recherche en tourisme

\section{Vers une professionnalisation et une industrialisation des activités dans le domaine du tourisme}

\section{Vincent Sabourin}

Volume 17, numéro 3, automne 1998

Pratiques contemporaines de gestion dans le domaine du tourisme

URI : https://id.erudit.org/iderudit/1072131ar

DOI : https://doi.org/10.7202/1072131ar

Aller au sommaire du numéro

Éditeur(s)

Université du Québec à Montréal

ISSN

0712-8657 (imprimé)

1923-2705 (numérique)

Découvrir la revue

Citer ce document

Sabourin, V. (1998). Vers une professionnalisation et une industrialisation des activités dans le domaine du tourisme. Téoros, 17(3), 3-4.

https://doi.org/10.7202/1072131ar 


\section{VERS UNE PROFESSIONNALISATION ET UNE INDUSTRIALISATION DES ACTIVITÉS DANS LE DOMAINE DU TOURISME}

\section{Vincent Sabourin, rédacteur invité}

La dernière decennie a amené des transformations importantes dans le secteur du tourisme La complexite relative des phenomènes de marché et de concurrence et la hausse des exigences envers les gestionnaires de l'industrie touristique sont des phénomènes qui mettent en perspective le role important des pratiques contemporaines de gestion dans le domaine du tourisme.

Le tourisme continue de subir de profondes transformations et toute une série de bouleversements viennent en modifier le panorama ainsi que l'ensemble de son fonctionnement. Ce numéro spécial de Teoros, portant sur les pratiques contemporaines de gestion du tourisme, met en humière deux phénomènes particuliers: la professionnalisation de la gestion et l'industrialisation de plusieurs secteurs d'activites dans le domaine du tourisme.

En matière de gestion, on assiste à une première transformation incontoumable, celle de la professionnalisation de la gestion. Cette professionnalisation de la gestion a des incidences sur les principales fonctions de l'entreprise touristique et sur sa pérennité. De façon particulière, elle vient modifier la nature des principales fonctions de l'entreprise :

- La commercialisation et le marketing;

- La gestion des opérations:

- La gestion financière et comptable;

- La gestion des ressources humaines;

- La stratégie de l'entreprise dans son ensemble.

La transformation touche aussi les differents aspects du contexte stratégique dans lequel auve l'entreprise touristique. Que l'on parle de démographie, du contexte réglementaire ou socio-politique, ou du contexte économique ou technologique, il apparaît clairement dans chacun de ces environnements qu'une convergence de facteurs fait nattre des problematiques particulieres. Une des plus importantes demeure l'industrialisation des activites. En effet, plusieurs secteurs qui étaient jusqu'a présent relativement fragmentés s'appuient sur un mode de fonctionnement de moins en moins artisanal.

Que ce soit dans le secteur des lignes aériennes où la concurrence et la liberralisation des marchés ont pris leur plein envol, ou encore dans le secteur de l'hôtellerie où l'on assiste à l'emergence de multiples chaînes, ces transformations amè- nent, dans une majorité de cas, une modification des règles du jeu et remettent en question l'envergure de marché des entreprises qui auvrent dans une même région géographique. D'autress secteurs, comme celui de la restauration et des attractions touristiques, sont aussi en voie d'industrialisation.

De tels bouleversements amenent une industrialisation de plusieurs secteurs d'activités et nous mettent face à de nouvelles problématiques sur le plan de la gestion. C'est l'objet du présent numéro qui regroupe une série d'arlicles sous un thème commun, soit celui des pratiques contemporaines de gestion.

Ce numéro s'efforce de mettre en lumière les contours d'une problématique qui comporte de multiples facettes. Au caur des facteurs qui sont plus que jamais des eléments déterminants du succès des entreprises dans le domaine du tourisme, se trouve le service à la clientèle, Le premier article, de Lawrent Bourdeau, porte justement sur ce thème.

La différenciation et le positionnement des produits sont d'autres éléments importants liés a la professionnalisation et à l'industrialisation. Ils sont même devenus essenttiels dans un contexte de forte internatio- 
nalisation des marchés. Ainsi, l'article de Pascal Tremblay pose la problématique de la differenciation des produits et de leur positionnement.

L'article de Vincen Sabourin examine les stratégies concurrentielles des stations de ski dans une perspective nord-américaine. Il met en perspective l'industrialisation d'un secteur d'activites, d'une part, et la professionnalisation de la gestion, d'autre part. C'est l'émergence du paradigme de la gestion stratégique qui permet d'identifier les facteurs, sous le controble de la gestion de l'entreprise touristique, qui peuvent l'amener à influencer considérablement les phénomènes de marché et de concurrence.

Le cadre juridique est l'un des facteurs du contexte stratégique de l'entreprise qui joue un rôle déterminant ê exerce une infuence considérable sur ses activités. Dans cette optique, l'article de Louis Jolin met en lumiere les implications juridiques du statut d'entreprise des associations touristiques.

L'application des pratiques contemporaines de gestion s'exerce à l'aide d'un certain nombre d'approches dont une des plus importantes demeure la planification et la gestion stratégique des activités de l'entreprise touristique. C'est l'objet de l'article de Hawkins et Holtz, deux auteurs américains de Washington qui présentent un modele et une expérience de planification strategique en écotourisme.
Parmi les concepts fondamentaux qui ont trait aux nouvelles pratiques contemporaines de gestion dans la domaine du tourisme, on trouve le rôle clé des canaux de distribution. En effet, la distribution est devenue au cours des dernières années un des elléments moteurs du développement d'un grand nombre d'entreprises. Elle repose sur la mise en place de systemes complexes dont les stratégies d'offre de produits et de discrimination de prix présupposent wne compréhension fine des exigences des clients, que ceux-ci soient grossistes, agents ou consommateurs. C'est le thème de l'article de François Bédard qui identifie les résultats préliminaires d'une enquête effectuéc auprès des agents de voyage.

Enfin, l'article de Michel Balfet propose un essai d'identification important qui porte sur les problématiques de gestion liées à la spécificité des produits et des services touristiques. Cet essai s'efforce d'élaborer un cadre conceptuel permettant de mieux comprendre les fondements de la discipline que constitue maintenant le champ du fourisme.

Ce numéno se termine par un article de conjoncture de Bruno Sarrasin et Jean Slafford et un article hors theme de Marc Leblanc.

Bonne lecture!
Vincent Sabourin. qui signe aussi dans ce numéro l'article *Les stratégies concurrentielles des stations de ski : une perspective nordaméricaine s, est professeur de stratégie de gestion a l'École des sciences de la gestion de I'Universite du Quebec a Montreal. Il a publie de nombreux articles dans des revues scien. tifiques et des actes de colloques. Il a aussi éte consultant dans le domaine du tourisme pour divers secteurs: immobilier, hôtellerie, restauration et centrales de résenvation. II détient un Ph. D. en Strategic Management de I'Universite McGill. 